\title{
Nach dem Shareholder Value? Kapitalmarktorientierte Unternehmenssteuerung in der Krise
}

Die Diskussion über die Finanz- und Wirtschaftskrise kreist gegenwärtig vor allem um die Re-Regulierung internationaler Finanzmärkte. Im Fokus stehen spezifische Finanzprodukte, Akteure und Regelwerke. In dieser Debatte geht jedoch oft verloren, dass sich hinter dem Begriff Finanzmarktkapitalismus ein ungleich komplexeres System verbirgt, das über zahlreiche Transfermechanismen mit der Realwirtschaft verzahnt ist. Wie kaum ein anderes Konzept steht der Shareholder Value für tief greifende Veränderungen in der Corporate Governance von Unternehmen. Er ist zum Synonym für die Auflösung der Deutschland AG und die Annäherung des rheinischkooperativen Kapitalismusmodells an seine angelsächsische Konkurrenzvariante geworden.

\section{1 \\ Einleitung}

Sehen Befürworter in der kapitalmarktorientierten Steuerung noch immer eine alternativlose Anpassung an eine globalisierte Wirtschaft (Merz 2008), halten Kritiker dies für eine höchst problematische Weichenstellung, die der Jagd nach kurzfristigem Gewinn Tür und Tor geöffnet hat (Urban 2009, S. 31ff.). So kontrovers die jeweiligen Positionen auch sein mögen Einigkeit besteht darin, dass die Shareholder-Value-Steuerung die Unternehmen, die Mitbestimmung und ihre Akteure gravierend verändert hat. In solchen Einschätzungen schwingt die Annahme mit, es handele sich um ein Set verbindlicher Organisationsformen und Praktiken, die, einmal implementiert, die soziale Realität in und im Umfeld von Unternehmen transformieren. Hier setzt der nachfolgende Beitrag an. Er will den Blick auf ein Phänomen lenken, das bislang viel zu wenig Beachtung gefunden hat. Hinter dem Konzept des Shareholder Value verbergen sich heterogene, gelegentlich durchaus widersprüchliche und vor allem beständig im Wandel befindliche Managementpraktiken. Ihnen ist gemeinsam, dass die Kapitalmarktorientierung als strategische Option, als bargaining chip und Legitimationsinstrument genutzt wird, um re-kommodifizierende Managementpolitiken durchzusetzen. An der Stoßrichtung solcher Praktiken wird sich - trotz globaler Finanz- und Überakkumulationskrise - wenig verändern, es sei denn, es finden sich soziale Akteure, die eine
Delegitimierung dieser Steuerungsformen arbeitspolitisch bewusst vorantreiben. Zur Begründung dieser Sichtweise wird zunächst (Abschnitt 2) das Konzept des Shareholder Value eingeführt und im Kontext unterschiedlicher wissenschaftlicher Ansätze beleuchtet. Anschließend (Abschnitt 3) geht es vor dem Hintergrund eines eigenen Ansatzes um die Durchsetzung der Shareholder-Steuerung und im Abschnitt 4 um deren Wandel im Gefolge der globalen Wirtschaftskrise. Abschnitt 5 lotet Möglichkeiten für arbeitspolitische Interventionen aus.

\section{2 \\ Was ist und wozu dient der Shareholder Value?}

In der arbeitspolitischen Debatte löst der Begriff Shareholder-Value-Steuerung Assoziationen zu einem Management der kurzen Frist und zu einer Kultur der Maximalprofite aus. Das Konzept selbst verheißt allerdings das genaue Gegenteil. Danach stellt die Stärkung der Eigentümerinteressen eine angemessene Lösung des Prinzipal-Agent-Problems der Unternehmenssteuerung dar. Dem Managerkapitalismus der fordistischen Ära wird der Kampf angesagt; die Orientierung an den Interessen der Kapitalmarktakteure soll die Autonomisierung des Top-Managements eindämmen und längerfristig für eine größere Effizienz der Unternehmenssteuerung sorgen (Rappaport 1986). Damit entspricht die Shareholder-Value-Doktrin passgenau einer zentralen Annahme der zeitgenössi- schen Marktorthodoxie. Aus deren Perspektive stellt das Ideal eines Unternehmers mit sozialer Verantwortung eine besonders problematische Verzerrung des Wettbewerbsgedankens dar (Friedman 1982, S. 165). Was der ideologische Marktfundamentalismus allgemein vorgibt, sucht die Shareholder-Value-Bewegung auf der Ebene der Unternehmenssteuerung umzusetzen. Eine strikte Ausrichtung an Eigentümerinteressen soll den „Opportunismus" des strategiefähigen Managements, dessen Neigung, Partialinteressen zulasten des Unternehmens zu verfolgen, wirksam und nachhaltig begrenzen.

\subsection{LOGISCHE KONTROLLPROBLEME}

In früheren Arbeiten (Dörre/Brinkmann 2005, S. 58ff.) haben wir bereits auf den logischen Konstruktionsfehler dieses Kontrollversprechens hingewiesen. Faktisch läuft die Eigentümerorientierung darauf

Klaus Dörre, Dr., Professor für Arbeits-, In-
dustrie- und Wirtschaftssoziologie an der
Friedrich-Schiller-Universität Jena. Arbeits-
schwerpunkte: Prekäre Beschäftigung,
Strategic Unionism, Kapitalismustheorien.
e-mail: klaus.doerre@uni-jena.de
Hajo Holst, Wissenschaftlicher Mitarbeiter
an der Friedrich-Schiller-Universität Jena,
Institut für Soziologie, Lehrstuhl für Arbeits-,
Industrie- und Wirtschaftssoziologie.
Arbeitsschwerpunkte: International
vergleichende industrielle Beziehungen,
nicht-standardisierte Beschäftigungsformen,
betriebliche Flexibilisierungsstrategien.
e-mail: hajo.holst@uni-jena.de

Klaus Dörre, Dr., Professor für Arbeits-, Industrie- und Wirtschaftssoziologie an der Friedrich-Schiller-Universität Jena. Arbeitsschwerpunkte: Prekäre Beschäftigung, Strategic Unionism, Kapitalismustheorien. e-mail: klaus.doerre@uni-jena.de Hajo Holst, Wissenschaftlicher Mitarbeiter ander Friedrich-Schiller-Universität Jena, Industrie- und Wirtschaftssoziologie. Arbeitsschwerpunkte: International verg/eichende industrielle Beziehungen, betriebliche Flexibilisierungsstrategien. e-mail: hajo.holst@uni-jena.de 
hinaus, die evolutionäre Vergesellschaftung der Unternehmensentscheidungen, die mit dem ausgehandelten, mitbestimmten Unternehmen erreicht wurde, sukzessive zurückzunehmen und sie im Namen der Liquidität einer auf bornierte Einzelinteressen gegründeten externen Kontrolle zu unterwerfen (Aglietta/Rebérioux 2005). Das ausgehandelte, mitbestimmte Unternehmen eines Kapitalismus mit koordinierten Märkten lässt sich hinreichend weder als Rechtsobjekt von Eigentümern noch als Bündelung formaler Vertragsbeziehungen begreifen. Faktisch ist dieses Unternehmen in seinen Legitimationen nach innen wie auch gegenüber seiner Umwelt längst zu einer öffentlichen Institution geworden. Im Idealfall stellt es eine annähernde Balance zwischen den Interessen verschiedener Stakeholder-Gruppen her und leistet zugleich eine Internalisierung gesellschaftlicher Kompromissbildungen.

$\mathrm{Zu}$ den Prämissen der ShareholderValue-Bewegung (Hiss 2009) gehört, dass die Kompromissbildungen in mitbestimmten Unternehmen $\mathrm{zu}$ schwerfällig und $\mathrm{zu}$ komplex seien, als dass sie in der Beschleunigungsspirale eines globalisierten Kapitalismus Bestand haben könnten. Eine straffere Ausrichtung der Unternehmensführung an Aktionärsinteressen soll die Effizienz der Unternehmensaktivitäten erhöhen und so zu Erträgen führen, die letztlich allen beteiligten Gruppen zugute kommen. Allerdings sind Aktionäre wie auch andere Kapitalmarktakteure zumindest in Hinblick auf die koordinierende Tätigkeit des Managements Outsider. Erfolg und Misserfolg der Managementtätigkeit können die Eigentümer im Grunde nur aus der Außenperspektive und ex post beurteilen. Gleiches gilt im Übrigen für Analysten und Rating-Agenturen, die mit in die Zukunft verlängerten Gegenwartstrends, mit Erwartungen und Erwartungserwartungen operieren.

Insofern führt die Shareholder-ValueDoktrin schon logisch-konzeptionell nicht zu einer Einschränkung der Macht des strategiefähigen Managements. Im Gegenteil, in dem Maße, wie die Eigentümerorientierung bewirkt, dass unternehmensinterne Kompromissbildungen ignoriert werden, schwindet der Einfluss von Insidern, die in der Lage wären, die Autonomisierung von Managementinteressen zu korrigieren. Anstatt das Top-Management an die kurze Leine zu legen, besteht vielmehr die Gefahr, dass die aktionärsorientierte Unterneh- mensführung (Fligstein 2001, S. 168f.) einer neuen Managerelite zum Durchbruch verhilft, die sich weder einem kollektiven Unternehmenswillen verpflichtet fühlt noch auf Wachstumsziele festlegen lässt, sondern stattdessen von einer hohen Übereinstimmung zwischen Eigen- und Unternehmensinteressen ausgeht und das Streben nach kurzfristiger Gewinnmaximierung ins Zentrum des eigenen Handelns stellt.

\subsection{KONKURRIERENDE DEUTUNGEN}

Die logische Inkonsistenz der ShareholderValue-Doktrin wird in der sozialwissenschaftlichen Beobachterperspektive auf unterschiedliche Weise reflektiert. Im Wesentlichen lassen sich zwei Gruppen von Ansätzen, strukturalistische und kulturalistische, unterscheiden. Für eher strukturalistisch argumentierende Ansätze ist die Shareholder-Value-Steuerung von Unternehmen konstitutives Element eines „Akkumulationsregimes der Vermögensbesitzer" (Aglietta 2000, S. 66) oder eines Finanzmarktkapitalismus (Windolf 2005, S. 20ff.), der sich seit den 1980er Jahren herausgebildet hat. Unter den Bedingungen unsicherer Märkte und der relativen Autonomisierung des Finanzsektors macht es für das Management weltmarktorientierter Unternehmen Sinn, strategische Operationen an den Renditen der marktführenden Unternehmen auszurichten. Eine Eigenkapitalrendite der Deutschen Bank von $25 \%$ rechtfertigt sich in der finanzkapitalistischen Logik, weil andere Kreditinstitute mit ihren Margen über die $30 \%$ gehen. In einer Ökonomie, in der Unternehmen $\mathrm{zu}$ Waren werden und überschüssigem Kapital als Anlageobjekt dienen, wird Liquidität zu einem strategischen Ziel des Top-Managements.

Um selbst akquisitionsfähig zu sein, aber auch, um sich vor feindlichen Übernahmen zu schützen, soll die Schwierigkeit, Liquidität zu generieren, überwunden werden. Dies geschieht, indem Unternehmen nach kaskadenförmig auf die dezentralen Einheiten heruntergebrochenen Renditeerwartungen oder Gewinnvorgaben geführt werden. Auf diese Weise unterliegt im Idealfall jede dezentrale Einheit einem strengen Finanzcontrolling. Finanzielle Operationen, selbst in kleineren Größenordnungen, lassen sich aus einer kontrollierenden Zentrale beobachten, die zudem in kurzen Zeitabständen Ergebnisberichte seitens der untergeordneten Hierarchie- ebenen einfordert. Während die frühere Manageraufsicht an einer langfristigen Stabilität der Kapitalakkumulation interessiert war, besteht das Kontrollregime des Shareholder Value ,auf den Leistungskriterien der Finanzmärkte“. Es zwingt „die Unternehmen zur höchstmöglichen kurzfristigen Kapitalbildung an der Börse“, und die allgegenwärtige Drohung, „das Management auszutauschen oder aufgekauft zu werden“, genügt, um immer wieder Druck auf die Lohnkosten und die Beschäftigung auszuüben, „ohne die geringste Rücksicht auf die zukünftige Entwicklung zu nehmen" (Aglietta 2000, S. 50).

Die in strukturalistischen Ansätzen unterstellte Funktionalität der ShareholderValue-Steuerung wird von stärker kulturalistisch argumentierenden Autoren kritisiert, die bezweifeln, dass sich realwirtschaftliche Unternehmensstrategien von Leistungskriterien der Finanzmärkte ableiten lassen. Schon aufgrund der Mehrdeutigkeit von Eigentümerinteressen gilt die in Grenzen deutungsoffene „Finanzmarktrationalität" als „eine Form wirtschaftlicher Rationalität unter anderen “ (Kädtler 2009, S. 7). Sie setzt sich in den Unternehmen niemals vollständig durch, weil die Produktion von Gütern und Dienstleistungen nicht ausschließlich und häufig nicht einmal primär finanzkapitalistischen Rationalitätskriterien entsprechen kann. Nach solchen Interpretationen handelt es sich bei der Shareholder-Value-Steuerung um einen, gleichwohl folgenreichen, „Rationalitätsmythos" (Deutschmann 1997), der allerdings weit über seinen ursprünglichen Entstehungskontext (weltmarktorientierte Aktiengesellschaften) hinaus ausstrahlen kann (zum KMU-Sektor: Becker 2009). Der „Druck“, der von institutionellen Anlegern und anderen Kapitalmarktakteuren ausgeht, wirkt nicht automatisch und vor allem nicht gleichförmig auf alle Unternehmen, er ist immer auch mikropolitisch hergestellt (Brinkmann 2009). Dennoch strukturiert er die Interaktionen maßgeblicher Akteure innerhalb wie außerhalb der Unternehmen.

\section{3 \\ Shareholder Value und neue Landnahme}

Wir sind der Ansicht, dass sich beide Interpretationsschemata weniger konträr zuein- 
ander verhalten, als es auf den ersten Blick scheinen mag. Neigen strukturalistische Ansätze bisweilen zu einem allzu simplen Funktionalismus, unterschätzen kulturalistische oder wissenssoziologische Deutungen die strukturbildenden Wirkungen der Shareholder-Value-Steuerung. Um die Stärken beider Betrachtungsweisen nutzbar machen zu können, definieren wir kapitalmarktorientierte Steuerungsformen nachfolgend als Transfermechanismus finanzkapitalistischer Landnahmen.

\subsection{EIN TRANSFERMECHANISMUS}

An dieser Stelle kann das Theorem kapitalistischer Landnahmen nicht umfassend erläutert werden. Im Kern besagt es, dass der Kapitalismus sich nicht aus sich selbst heraus reproduzieren kann. Er bleibt darauf angewiesen, „äußere Märkte“ zu nutzen, die er sowohl in nichtkapitalistischen Gesellschaften als auch in nicht kommerzialisierten Sektoren innerhalb kapitalistischer Gesellschaften findet. Im Unterschied zu klassischen Landnahmetheorien (Luxemburg 1975; Arendt 2006) gehen neuere Versionen davon aus, dass ein „Außen“ der kapitalistischen Produktionsweise auch aktiv hergestellt werden kann (Harvey 2005, S. 140). Aktive Herstellung bedeutet, dass die Kette der Landnahmen prinzipiell unendlich ist. Der „Sündenfall“ einer „Sprengung rein ökonomischer Gesetzmäßigkeit durch politisches Handeln" (Arendt 2006, S. 335) kann und muss sich folgerichtig auf erweiterter Stufenleiter beständig wiederholen. Dabei lassen sich zwei Grundformen der Landnahme unterscheiden (Dörre 2009, S. 42ff.). Die eine beruht auf DeKommodifizierung, d. h. auf langfristigen Infrastrukturinvestitionen in den sekundären oder tertiären Kapitalkreislauf sowie auf Formen der Staatsintervention, die die Lohnarbeit sukzessive von Marktrisiken abkoppeln. Die fordistische Landnahme nach 1945 entsprach diesem Typus. Mit organisierten Arbeitsmärkten, dem Ausbau kollektiver Sicherungssysteme, Staatsunternehmen und einem expandierenden öffentlichen Dienst entstand eine gemischte Wirtschaft, in der ganze Bereiche der privaten Verwertung weitgehend entzogen waren. Erstmals in der Geschichte bildete sich ein Kapitalismus ohne sichtbare industrielle Reservearmee heraus (Lutz 1984, S. 186ff.). Sozial geschützte Lohnarbeit verwandelte sich in eine gesellschaftliche Integrationsmaschine und schuf auch für einen
Großteil der Lohnabhängigen erstmals die Voraussetzungen für eine längerfristige Lebensplanung.

Die finanzkapitalistische Landnahme, wie sie seit den 1980er Jahren alle kapitalistischen Zentren und zeitversetzt auch die Länder des vormals staatsbürokratischen Sozialismus erfasst hat, entspricht einer anderen Grundvariante. Dieser zweite Typus stellt ein „Außen“ über Re-Kommodifizierung und sozialen Ausschluss her. Im Kern zielt die finanzkapitalistische Landnahme auf Institutionen, gesellschaftliche Organisationsformen und soziale Regeln, die die Wirkungen marktförmiger Koordinationsmechanismen begrenzen. Der Modus operandi dieser Landnahme umfasst eine Vielzahl an Transfermechanismen, zu denen auch die Shareholder-Value-Steuerung gehört. Das Shareholder-Value-Regime fungiert gewissermaßen als Bindeglied zwischen den Anforderungen sich rasch verändernder Märkte und den Profitabilitätsinteressen der Eigentümer einerseits sowie der Unternehmens-, Betriebs- und Arbeitsorganisation andererseits. Dabei muss systematisch zwischen dem (1) Leitbild des Shareholder Value, seiner (2) praktischen Umsetzung sowie (3) den Wirkungen unterschieden werden, die dieses Kontrollregime auf soziale Regeln ausübt.

(ad 1) Die strukturierende Idee des Leitbilds besteht darin, die Profitabilität des Unternehmens zur zentralen Steuerungsgröße zu erklären, an der sich andere Leistungskriterien auszurichten haben. Shareholder-Value-Steuerung bedeutet, dass unabhängig von den konkreten Steuerungspraktiken eine Art Mindestprofitabilität definiert wird, die es unter optimaler Ausnutzung interner wie externer Flexibilisierungsinstrumente $\mathrm{zu}$ gewährleisten gilt. In diesem System erscheinen der Gewinn und abgeleitet davon die Rendite nicht mehr als Resultat real erbrachter ökonomischer Leistungen; vielmehr werden Gewinnmargen oder Ergebniserwartungen als Planungsgröße vorausgesetzt und in Gestalt von Kennziffern und Budgetierung zu einer verbindlichen Zielgröße auch dezentraler Einheiten erklärt. Dies kann z. B. geschehen, indem Unternehmen samt Immobilien und Anlagen als Ansammlung von Vermögenstiteln betrachtet werden, um sodann die Eigenkapitalrentabilität jedes Titels zu bewerten. Zentrale Steuerungsgröße ist in solchen Fällen der „Geschäftwertbeitrag“, der „EBIT“ oder Ähnliches, also ein Steuerungskriterium, dessen
Funktion darin besteht, die Mindestprofitabilität sichtbar zu machen. Dies vor Augen, lässt sich präzisieren, worin die InnenAußen-Dialektik kapitalmarktorientierter Steuerungsformen wurzelt. Das „Außen“ dieser Steuerungsform sind nicht Realwirtschaft und Unternehmen schlechthin, vielmehr wird das „Außen“ von jenen Institutionen, Organisationsformen und sozialen Regeln des mitbestimmten Unternehmens gebildet, die in der Blütezeit des fordistischen Kapitalismus die Kongruenz zwischen der längerfristigen Kapitalakkumulation und der Stabilität des Lohnverhältnisses sichergestellt und so für eine fortschreitende De-Kommodifizierung von Lohnarbeit gesorgt hatten.

(ad 2) Die praktische Umsetzung des Leitbilds vollzieht sich allerdings in Unternehmen, die sich in Anlehnung an die Feldkonzeption Pierre Bourdieus (1998) als tief gestaffelte Machtfelder begreifen lassen. Märkte sind Felder der Unternehmen. Ihre Besonderheit resultiert aus Machtrelationen und Spielregeln, welche neben dem Staat wesentlich von dominanten industriellen Komplexen bestimmt werden. Erscheint das Unternehmen aus der makroökonomischen Perspektive als homogener Akteur, verändert sich der Blickwinkel, sobald das Feld des Unternehmens betrachtet wird, das eine relative Autonomie gegenüber den aus dem Feld der Unternehmen herrührenden Zwänge besitzt. Ähnliches gilt für das Feld des Betriebs oder das Feld des Profitcenters. Auf jeder dieser Ebenen existieren besondere Interessen, Machtkonstellationen und Spielregeln, die sich nicht im Durchgriff eines neuen Steuerungssystems von heute auf morgen grundlegend verändern lassen. So sind z. B. die Interessen der Unternehmenszentrale andere als die der Sparte, und deren Interessenlagen unterscheiden sich wiederum von denen betrieblicher Geschäftsleitungen. Schon innerhalb der Managementhierarchie existieren somit diverse Filter, die im Grunde ausschließen, dass sich ein Steuerungssystem in Reinform durchsetzt.

(ad 3) Viel wahrscheinlicher ist, dass jede neue Steuerungsform im Verlauf ihrer praktischen Durchsetzung erhebliche Modifikationen erfährt. Ältere, eingespielte Steuerungssysteme werden nicht einfach ersetzt, sondern mit neuen Elementen kombiniert und umgeformt. Ähnliches gilt im Übrigen auch für die Auswirkungen der Shareholder-Value-Steuerung auf marktbegrenzende soziale Regeln. Es ist in höchs- 
tem Maße unwahrscheinlich, dass solche Regelwerke und ihre Institutionalisierungen gewissermaßen im Sturmangriff geschleift und beseitigt werden. Realitätsnäher ist die Vorstellung eines graduellen Wandels (Streeck 2009, S. 240), der sich innerhalb wie unterhalb scheinbar intakter Institutionen bemerkbar macht. Noch immer gibt es das ausgehandelte Unternehmen, interne Arbeitsmärkte, Mitbestimmungskulturen, arbeitsrechtliche Bestimmungen und Kollektivverträge. Doch in den alltäglichen Erfahrungen erheblicher Teile der Beschäftigten büßen diese Institutionen und Regelwerke sukzessive ihre Schutzfunktion ein. Sie verschwinden nicht, doch ihre Verankerung in den alltäglichen Praktiken und Kompromissbildungen maßgeblicher Akteure erodiert. Und mit den Spielregeln verändern sich zugleich die Felder, in denen diese Akteure agieren.

\subsection{DREI PHASEN DER DURCHSETZUNG}

Nutzt man die Heuristik ineinander geschachtelter, relativ autonomer Machtfelder, wird man die Vorstellung, dass es sich bei der Shareholder-Value-Steuerung um ein Set verbindlicher Managementprinzipien, Instrumente und Praktiken handelt, das in jeder Unternehmensorganisation nahezu gleich wirkt, rasch ad acta legen müssen. Realitätsnäher ist die Vorstellung einer Leitidee, die im Prozess ihrer Durchsetzung modifiziert und mit unterschiedlichen, sich immer wieder wandelnden Praktiken verknüpft wird. Exakt dies lässt sich zeigen, wenn man die Phasen der Durchsetzung und Anwendung des ShareholderValue-Konzepts in weltmarktorientierten Unternehmen betrachtet. Für die Zeitspanne zwischen 1990 und 2009 lassen sich drei Phasen unterscheiden.

Die erste Phase (1990 bis etwa 1997) beinhaltet die Durchsetzung von Elementen einer flexiblen Produktionsweise. ${ }^{1}$ Zwar existieren auch in dieser Zeit bereits Unternehmen, die nach Shareholder-Doktrin geführt werden. Dabei handelte es sich jedoch um Töchter von Konzernen mit Sitz im Ausland. Die Auswirkungen der straffen Profitsteuerung werden in dieser Phase durch die noch einigermaßen intakten korporativen Praktiken eines sozial-bürokratischen Kapitalismus abgefedert. Dennoch lassen sich bereits Konturen einer flexiblen Produktionsweise erkennen. Die entscheidenden Veränderungen vollziehen sich auf der Ebene der Unternehmens- und Betriebsorganisation. Das betriebswirtschaftliche Geheimnis der flexiblen Arbeitsweise wurzelt in einem break even point, der so fixiert ist, dass vergleichsweise große Konjunkturschwankungen aufgefangen werden können, ohne die Gewinnzone zu verlassen. In der flexiblen Organisation stellt die Belegschaft das entscheidende Flexibilitätspotenzial dar, mit dessen Hilfe eine Produktionsweise mit knappen Zeit-, Material- und Personalpuffern funktionsfähig gehalten wird. Inszenierte Marktbeziehungen sorgen für eine Intensivierung der Konkurrenz im Inneren der Firmenorganisation. Die Verstetigung des Wettbewerbs ist der entscheidende Anreiz für immer neue Anstrengungen zur Rationalisierung und Kostensenkung. Auf diese Weise bewirkt die flexible Firmenorganisation auch, dass Beschäftigte aktiv in Rationalisierungsprozesse einbezogen werden.

Erst in einer zweiten Phase (zwischen 1997-2002) folgt die Anwendung der Shareholder-Value-Doktrin in einer größeren Zahl weltmarkorientierter Unternehmen (vgl. Dörre 2002, S. 345ff.). Der Siemens-Konzern stellt dafür nur ein besonders markantes Beispiel dar, weil dem Übergang zur wertorientierten Steuerung hier eine Aktionärsrevolte vorausgegangen ist. Hauptmerkmal dieser Phase ist, dass eine an Kapitalmärkten orientierte Ergebnisbewertung eingeführt und auf die dezentralen Unternehmenseinheiten heruntergebrochen wird. In dieser Phase verengt sich der Korridor für arbeitsorganisatorische Modernisierungen. Unter dem Druck eines Managements der „kurzen Frist“ wird nur noch praktiziert, was sich unter diesem Regime machtpolitisch einigermaßen problemlos bewerkstelligen lässt. Gruppenarbeitsexperimente mit großen Tätigkeitsumfängen und Partizipationsspielräumen geraten in die Defensive. Dennoch darf die dynamische Wirkung des Shareholder-Regimes nicht unterschätzt werden. Mit seiner Hilfe können verkrustete Hierarchien und Führungsstrukturen aufgebrochen, Geschäftsfelder restrukturiert und Kostentreiber sichtbar gemacht werden. Über Entgeltsysteme und Boni-Regelungen werden nicht nur Unternehmensvorstände, sondern ganze Hierarchien bis in die dritte und vierte Ebene hinein auf Renditeerwartungen oder Gewinnvorgaben verpflichtet. Bei der Durchsetzung des neuen Steuerungssystems spielen Kontingenzen, Zufälle, Per- sonen und natürlich auch differente Produktionswelten eine große Rolle. Vielfach ist der Übergang zur wertorientierten Steuerung mit Wechseln in den Unternehmens- und Betriebsleitungen verbunden. Schon in dieser Phase, die keineswegs zufällig mit zahlreichen politischen Maßnahmen zur Deregulierung von Finanzbeziehungen einhergeht, vereinigt die Idee des Shareholder Value höchst unterschiedliche Managementpraktiken. Die Bandbreite reicht von der Steuerung über target costs (nur der Preis des Produkts wird von der Zentrale vorgegeben, die Arbeitsorganisation obliegt den dezentralen Einheiten) bis zu Kombinationen aus straffer Profitsteuerung und detaillierten Vorgaben für die Produktionskonzepte. Deutlich wird auch, dass Shareholder Value und interne Finanzialisierung andere Steuerungssysteme nicht einfach ersetzen, sondern sie auf neue Weise synchronisieren.

In der dritten Phase (2002-2006) entfaltet sich schließlich die regelverändernde Kraft des neuen Kontrollregimes in vollem Maße. ${ }^{2}$ Hier tritt deutlich zutage, was wir in früheren Untersuchungen unterschätzt hatten. Mit der Installierung des Shareholder-Value-Regimes geht es nicht mehr, wie noch in der ersten Phase, um Profitabilität an sich. Vielmehr ist die Sicherung von Mindestrenditen bzw. Mindestgewinnen ein wesentliches Managementziel. Dies führt in der Konsequenz dazu, dass selbst solche Werke und Betriebsteile in die Mühlen des Standortwettbewerbs geraten, die eigentlich gewinnbringend produzieren. Werksaufspaltungen, Ausgründungen und Auslagerungen sorgen dafür, dass unternehmensübergreifende Regelwerke an Verbindlichkeit verlieren. Dies ist nur ein Beispiel für die schleichende, graduelle, in der Summe gleichwohl regelverändernde Kraft des Shareholder-Value-Regimes. Formal bleiben Mitbestimmung und Kollektivvereinbarungen in Kraft. Mehr noch: Betriebsräte und betriebliches Management werden geradezu in Wettbewerbskoalitionen hineingezwungen; ihre gemeinsamen „Kontrahenten“-Unternehmenszentralen oder institutionelle Anleger - befinden sich

\footnotetext{
1 Vgl. dazu Dörre (2002) S. 37ff.; die Studie basiert auf Mehrfacherhebungen in 36 Betrieben vornehmlich aus der Metall- und Elektroindustrie sowie der IT-Branche.

2 Vgl. Dörre/Röttger (2006) S. 141ff.; erfasst wurden 23 Betriebe vornehmlich aus der Metall- und Elektroindustrie sowie der IT-Branche.
} 
nicht selten außerhalb der Landesgrenzen. Eine ständige Konkurrenzsituation unter und zwischen den Beschäftigten lässt die Schutzfunktion kollektiver Vereinbarung verblassen. Unterhalb einer fortexistierenden institutionellen Hülle entsteht so ein "Außen“, eine andere, vom institutionellen Rahmen abweichende soziale Realität.

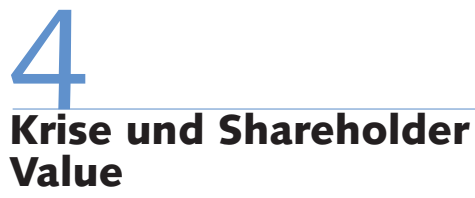

Halten wir fest: Schon vor der globalen Finanz- und Wirtschaftskrise bezeichnet der Terminus Shareholder-Value-Steuerung eine konzeptive Idee mit Leitbildfunktion, die im Zeitverlauf mit unterschiedlichen Praktiken kombiniert wird. Im Grunde handelt es sich um die Finanzialisierung einer flexibel-marktzentrierten Produktionsweise, deren Kernelemente bereits etabliert waren, bevor kapitalmarktorientierte Steuerungsformen eingeführt wurden.

\section{1 "OPPORTUNISMUS“ UND MODERNISIERUNG}

Das Shareholder-Regime konstituiert einen Möglichkeitsraum, den das strategiefähige Management für Praktiken nutzt, die nach Streeck (2009, S. 241) den „Opportunismus“, die „Nichtregierbarkeit" kapitalistischer Akteure ausmachen. Immer wieder finden sich Unternehmensleitungen, die gleichsam als "first movers" soziale Regeln unterlaufen, tarifliche Standards korrigieren und die Abweichungen teilweise in betrieblichen oder unternehmensweiten Wettbewerbspakten fixieren. Dabei wirkt die kapitalmarktorientierte Steuerung ähnlich wie die Globalisierungsmetapher und der mit ihr verknüpfte Standortwettbewerb. Schon die Antizipation einer möglichen feindlichen Übernahme des Unternehmens kann, vom Management als bargaining chip eingesetzt, genügen, um die Gegenseite zu Zugeständnissen zu bewegen. Lässt sich „opportunistisches“ Verhalten der Unternehmensleitungen, das in einem Spannungsverhältnis zu verbindlichen sozialen Regeln steht, an vielen Beispielen belegen, muss die Gleichsetzung dieses Verhaltens mit modernem, nichttraditionalistischem Handeln „par excellence“ (ebd.) mit einem Fragezeichen ver- sehen werden. Zwar hat das Shareholderregime teilweise zu größerer Kostentransparenz beigetragen und den ökonomischen Strukturwandel beschleunigt; seine Hauptwirkung ist jedoch eine andere. Es liefert den Begründungsrahmen und die Machtressourcen für Managementpolitiken, die Wettbewerbsvorteile primär über die ReKommodifizierung und teilweise über die Prekarisierung von Lohnarbeit zu realisieren suchen. Ein solcher Modus operandi kapitalistischer Landnahme entlastet das operative Management von aufwendigen Prozess- und Produktinnovationen.

Ein Entwicklungspfad, der den Lohn und die Arbeitsstandards in die Konkurrenz hineinnimmt und zugleich Schlüsselinnovationen - etwa die ökologische Modernisierung des Wertschöpfungssystems Automobil - aufschiebt, lässt sich allenfalls als regressive Modernisierung bezeichnen. Naheliegend ist, dass kapitalmarktorientierte Steuerungsformen, die die strategischen Weichenstellungen zugunsten dieses Pfades entscheidend begünstigt haben, an Akzeptanz verlieren, wenn ihre Dysfunktionalität in einer Krisensituation deutlich zutage tritt. Einseitig rendite- und kostenorientierte Managementstrategien haben Unternehmen hervorgebracht, die in der Krise weitreichender Staatsinterventionen bedürfen, um auch künftig noch am Markt bestehen zu können. In einer Ökonomie, in der staatliche Schutzschirme, Langfristkurzarbeit und Abwrackprämien notwendig sind, um zumindest die schlimmsten Krisenauswirkungen abzufedern, sollte ein Shareholder-Regime, das die Zone unkontrollierter Autonomie für das Top-Management eher vergrößert hat, seine Legitimationsgrundlage rasch einbüßen. Doch das ist offenkundig nicht der Fall. Ein Grund sind Selbsttransformationen des Regimes, die in manchen Konzernen schon deutlich vor Ausbruch der Krise eingesetzt haben. Obwohl es noch an einer differenzierten Überblicksempirie mangelt, lassen unsere Recherchen in ausgewählten Betrieben und Unternehmen doch erste Trends erkennen. ${ }^{3}$

\subsection{KRISE UND STEUERUNG - EIN FALLBEISPIEL}

Im Fall eines ehemaligen Nutzfahrzeugherstellers, der sich in den zurückliegenden Jahren in einen internen Zulieferer eines Automobilkonzerns verwandelt hat, ist das Leitbild des Shareholder Value in den Legi- timationsstrategien des Managements bereits seit 2006 mehr und mehr in den Hintergrund getreten. Entscheidende Ursache hierfür war ein Wechsel im Konzernvorstand. Die neue Führung grenzte sich explizit von der Shareholder-Value-Orientierung des alten Managements ab und verkündete eine neue „Philosophie“, deren Umsetzung zu gravierenden Veränderungen in der Unternehmens- und Betriebsorganisation geführt hat. Durch eine Re-Zentralisierung zuvor nach unten delegierter Verantwortlichkeiten wurde die CenterStruktur, die mit der internen Finanzialisierung verknüpft war, teilweise zurückgenommen. Zwar sind die Abteilungsleiter in den Betrieben weiterhin ergebnisverantwortlich und ihre Bezahlung ist nach wie vor an das Geschäftsergebnis gekoppelt, die Entscheidungsbefugnisse der dezentralen Ebene sind jedoch deutlich beschnitten worden. Die Konzernzentrale steuert über Budgets und die Personalplanung; beim Spartenmanagement ist die Produktionsplanung zentralisiert. Am Beispiel der Personalpolitik wird der Autonomieverlust des betrieblichen Managements besonders deutlich: Schon vor der Krise mussten Festeinstellungen von der Konzernzentrale genehmigt werden. Selbst die Standortleitung konnte solche Personalentscheidungen nicht autonom treffen. In der Krise ist der Abbau flexibel Beschäftigter nun zur Ultima ratio des Managements geworden. Die Konzernzentrale hat entschieden, dass die Verträge der Leiharbeiter und befristet Beschäftigten nicht verlängert werden; das betriebliche Management muss dem Folge leisten.

Daran zeigt sich, dass im Betrieb mehrere Systeme ineinandergreifen: die zentrale Steuerung durch den Konzern, die Steuerung der Abteilungen innerhalb der Sparte sowie die werksinterne Steuerung. Vor allem die operativen Abteilungen agieren mit drei einander überlappenden Steuerungssystemen, welche faktisch in der - von der Budgetsteuerung und der Produktionsplanung gerahmten - Ergebnisverantwortung der Abteilungsleiter zusammenlaufen. Die Abteilungsleiter befinden sich an der Schnittstelle; sie müssen die kapital-

\footnotetext{
3 Die folgenden Ausführungen beruhen auf Holst et al. (2009) mit Recherchen in zwölf Betrieben sowie auf Untersuchungen in einem Werk der Automobilindustrie (mehr als 50 Interviews im Oktober 2009), das in Vorläuferprojekten mehrfach untersucht wurde (vgl. Dörre 2002, Fall E).
} 
marktorientierten mit den produktionsbasierten Zielen der Unternehmenssteuerung vermitteln. Im Werk existiert somit nach wie vor kein kohärentes Steuerungssystem, das von oben nach unten in die Produktion durchgreift. Der Transfermechanismus, mit dem die Kapitalmarktorientierung der Konzernführung auf die Produktionssphäre Einfluss nimmt, setzt sich aus den beiden Instrumenten Budgetsteuerung und Personalplanung zusammen. Die permanent erhobenen Kennzahlen in Gestalt der sogenannte KPIs (Key Performance Indicators) fungieren als ein zusätzliches Kontroll- und Überwachungsinstrument der Führungsebene.

Insgesamt belegt das Beispiel einen interessanten Wandel des unternehmensinternen Steuerungsmodells. Wenngleich der Shareholder Value als Begründungsfolie für Managementstrategien in den Hintergrund tritt und einer neuen „Mode“ weicht, werden in Gestalt der Budgetierung und der zentralen Personalplanung Elemente einer kapitalmarktkonformen Konzernsteuerung noch stärker als zuvor in den Abläufen verankert. Die Abteilungsleiter haben zwar Ergebnisverantwortung, werden aber von der Zentrale über Budgets gesteuert. Von der Budgetierung erfasst sind auch die Personalausgaben, und genau dies erzeugt Druck auf die Beschäftigten und ihre Interessenvertretungen. In Phasen anziehender Konjunktur sind die Verantwortlichen nach Ausschöpfung des Personalkontingents gezwungen, auf Leiharbeiter auszuweichen, weil diese als Sachkosten geführt werden können. Wie viele andere Einheiten weltmarktorientierter Unternehmen operiert auch der Fallbetrieb mit der Vorgabe, einen festen Bestandteil der Belegschaft flexibel zu halten. Dies ist die Voraussetzung, um - wie geschehen - im Krisenfall rasch Personalpuffer abzuschmelzen.

Was wir im untersuchten Werk in einer Krisensituation antreffen, ist gewissermaßen eine kapitalmarktkonforme, hoch flexible, kurzfristorientierte Steuerungsform ohne Shareholder-Value-Semantik. Als Leitbild spielt der Shareholder Value nur noch eine nachrangige Rolle. Gleichzeitig lebt die Substanz des an kurzfristigen Gewinn- bzw. Renditezielen orientierten Kontrollregimes jedoch fort. Zwar haben Gewinnvorgaben in der Krisensituation nur eine geringe Bedeutung; an der Kurzfristorientierung der Steuerung hat sich jedoch nichts geändert. Die Ergebnisse werden noch immer in kurzen Zeitabständen bewertet. In der Krise gilt das Motto „Ergebnis retten!“ Um die Liquidität zu erhöhen und den Abfluss finanzieller Mittel $\mathrm{zu}$ verhindern, werden in allen Bereichen kurzfristige Einsparmöglichkeiten gesucht und auch ausgenutzt, selbst wenn dadurch die Stabilität der Produktionsprozesse gefährdet wird. Das zeitweise Abstellen von Maschinen, um Strom zu sparen, erhöht ebenso die Ausfallwahrscheinlichkeit wie das Zurückstellen notwendiger Investitionen. An diesen Beispielen wird die Durchsetzungsmacht und Beharrungskraft der Kapitalmarktorientierung in der Unternehmensführung auch und vor allem in der gegenwärtigen Krisensituation deutlich: Langfristige Produktionsinteressen müssen hinter kurzfristigen Liquiditätsinteressen zurückstehen.

Auch der Druck auf die Beschäftigten und ihre Interessenvertretungen lässt nicht nach. Im Gegenteil: Innerhalb des Konzerns findet ein „Flexibilitätswettbewerb“ statt, in welchem die Anpassungsbereitschaft der Belegschaften zur entscheidenden Ressource geworden ist. Ein Teil der „überzähligen“ Arbeitskräfte wird an die wenigen Standorte verschoben, die aufgrund des zentral durchgesetzten Abbaus von Leiharbeitern und befristet Beschäftigten Personalbedarf haben. Für die Standorte mit Arbeitskräfteüberhang sind die konzerninternen Personalverschiebungen lukrativ, da sie kurzfristig die Personalkosten reduzieren und damit das Quartalsergebnis verbessern. Standorte, denen es mit Unterstützung der Interessenvertretungen gelingt, ihren Belegschaften diese - mit erheblichen Belastungen verbundenen - Flexibilitätsleistungen abzuringen, stehen im Standortwettbewerb vergleichsweise gut da. Kurzfristig wirkt sich die Flexibilität der Belegschaften positiv aus, weil auf diese Weise Beschäftigung gesichert werden kann, langfristig besteht jedoch die Gefahr, dass sich die Standorte und damit die Angestellten bei den Leistungsstandards wechselseitig "nach oben konkurrieren“. Um die finanziellen Vorteile der Kurzarbeit optimal auszunutzen, versuchen einzelne Abteilungen trotz der geringeren Zahl von Beschäftigten an weniger Arbeitstagen einen stabilen Output zu erreichen. Die Betriebe externalisieren auf diese Weise einen Teil der Produktionskosten, für die Beschäftigten sind Leistungsintensivierung und Arbeitsverdichtung die Folge.

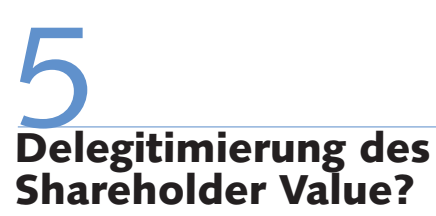

Vorerst ist unklar, ob und in welchem Maße sich dieses Beispiel verallgemeinern lässt. Aus parallelen Untersuchungen (Holst et al. 2009) wissen wir, dass eine Planwirtschaft im Dienste stabiler Gewinnmargen und Renditen, die mittels Budgetierung und Headcount (Personalvorgaben) „regiert", auch in anderen Unternehmen eine unhintergehbare Realität ist. Dies weist auf ein Phänomen hin, das wir zu Beginn unserer Studien so nicht erwartet hatten. Fakt ist nicht nur, dass die Leitidee des Shareholder Value mit höchst unterschiedlichen, sich ständig im Fluss befindlichen Praktiken kombiniert werden kann. Möglich ist auch, dass die Essenz dieses Kontrollregimes, eine marktgetriebene und an Kurzfristzielen orientierte Ergebnissteuerung, fortexistiert, ohne unter der alten Leitidee des Shareholder Value zu firmieren. Möglicherweise muss gar von einer Verfestigung kapitalmarktorientierter Steuerungsformen ausgegangen werden, die losgelöst von Shareholder-ValueAnsätzen realisiert wird. Denn Budgetierung und verbindliche Personalkennziffern stellen machtvolle Hebel der Zentralen dar, mit deren Hilfe die Produktion zumindest punktuell auf kapitalmarktkonforme Ziele hin ausgerichtet werde kann.

Sollte dies in größerem Maßstab zutreffen, stehen alternative arbeitspolitische Ansätze vor einem Problem. Es mag durchaus richtig sein, die „Herrschaft des Shareholder-Value-Regimes“ (Urban 2009, S. 34) $\mathrm{zu}$ attackieren; wir selbst haben für eine solche Strategie plädiert (Dörre et al. 2009, S. 59ff.). Doch die arbeitspolitische Attacke wird schwierig, wenn gar nicht klar ist, gegen welche Praktiken genau sie sich zu richten hat. Das Managementkonzept des Shareholder Value kann verschleißen, ohne dass dies notwendig mit einem Bedeutungsverlust wertorientierter Steuerungskriterien einhergehen muss. Auch die Konkurrenz unter den Beschäftigten kann weiter angeheizt und der Druck auf tarifliche Normen noch gesteigert werden, obgleich das Regime des Shareholder Value verblasst. Aus diesem Grund benötigen arbeitspolitische Gegenstrategien ein eigenes Leitbild, eine strukturierende Idee, die auch diskursiv einen Kontrapunkt setzt. Dabei 
gilt es zu berücksichtigen, dass die Shareholder-Value-Steuerung wie ihre potenziellen Nachfolger als Transfermechanismen eines finanzkapitalistischen Systems fungieren, das die Realwirtschaft permanent mit Rendite- und Gewinnerwartungen konfrontiert, die allein über Produktivitätszuwächse gar nicht $\mathrm{zu}$ befriedigen sind. Das ist der Grund, weshalb die finanzgetriebene Landnahme einem Steigerungsprinzip folgt: Sie verlangt beständig nach neuem „Land“, nach neuen, billigen Vermögenswerten, weiteren Privatisierungen, nach größerer Flexibilität in Unternehmen und am Arbeitsmarkt und nicht zuletzt nach Arbeitskraft, deren Preis unter ihren Wert gedrückt wird.

Soll erfolgreich gegengesteuert werden, kann dies nicht allein auf der Ebene des Unternehmens oder des Betriebs geschehen. Gefragt ist ein Gegenentwurf, der die systemische Dimension der finanzkapitalistischen Landnahme ins Visier nimmt. In diesem Zusammenhang fällt die erstaunliche Karriere auf, die der vermeintlich angestaubte Begriff der Wirtschaftsdemokratie in jüngster Zeit erfahren hat. Selbstverständlich kann es nicht darum gehen, ältere sozialdemokratische (Fritz Naphtali) oder linkssozialistische (Viktor Agartz, Ota $S i k)$ Konzeptionen einfach zu beerben. Allerdings wirft die Krise des Finanzkapitalismus von sich aus die Frage nach neuen Formen von Wirtschaftsdemokratie auf. In diversen „Managementphilosophien“ ist es inzwischen ein Gemeinplatz, dass Rationalisierungs- und Innovationsprozesse auf das informelle Wissen von Beschäftigten angewiesen sind. Was auf der Unternehmensebene eingeklagt, wenngleich selten mit Konsequenz praktiziert wird, kann in der Gesellschaft, bei den Auseinandersetzungen um das „Was“ und „Wozu“ von Produktion und Konsum aber wohl kaum falsch sein. Das zumal, wenn etwa beim Management der Banken- oder der Autokrise, längst Planungsmechanismen greifen, die freilich jenseits demokratischer Öffentlichkeiten von den gleichen Expertokratien bedient werden, deren Definitionsmacht das Desaster auf den Weltfinanzmärkten mit verursacht hat.

Wirtschaftsdemokratische Ansätze, die das Definitionsmonopol solcher Expertokratien aufbrechen wollen, müssten auf mindestens vier Ebenen etabliert werden: als direkte Partizipation von Beschäftigten am Arbeitsplatz, im Betrieb und auch in den Interessenorganisationen; als institutionalisierter Kollektivwille innerhalb und im Umfeld vor allem großer Unternehmen, der wieder explizit erfahrbar macht, dass es sich bei diesen Wirtschaftsorganisationen um öffentliche Institutionen handelt; über regionale wie nationale Wirtschafts- und Strukturräte sowie als Demokratisierung wichtiger internationaler Institutionen. Letzteres hieße in ersten Schritten Europäisierung der Mitbestimmung, aber auch selbstkritischer Umgang mit korporatistischem Filz in den Interessenvertretungen und darüber hinaus die Beteiligung von Repräsentanten reproduktiver Interessen (Umwelt- und Konsumentenorganisationen, NGOs) an wichtigen Unternehmensentscheidungen. Die Ausweitung demokratischer Partizipation auf strategische Investitionsentscheidungen großer Unternehmen wäre gut in ein solches Programm zu integrieren. Solche Ansätze könnten zudem mit einer arbeitspolitischen Perspektive verbunden werden, die die workforce von Wirtschaftsorganisationen als Ganzes in den Blick nimmt. Vieles, was derzeit als unübersichtliche Gemengelage erscheint das unverbundene Nebeneinander von Arbeitskraftunternehmer, immaterieller Arbeit, flexiblem Taylorismus, standardi- sierter Dienstleistungsarbeit und prekärer Beschäftigung -, ließe sich mit arbeitspolitischen Strategien angehen, die die Gesamtarbeit in Unternehmen oder Wertschöpfungssystemen betrachten. Auf diese Weise wäre - zumal in transnationalen Wertschöpfungssystemen - auch das „Außen“ sozial geschützter Lohnarbeit, wären die verschiedenen Formen prekärer und informeller Arbeit in eine wirtschaftsdemokratisch fundierte Gegenstrategie zu integrieren.

Freilich liegen auch die Einwände gegen einen solch systemischen arbeitspolitischen Ansatz auf der Hand: Macht es in einer Zeit, in der Betriebsräte und Gewerkschaften vor allem Abwehrkämpfe zu führen haben, um ,industrielle Kerne zu sichern" (Huber 2009), überhaupt Sinn, nach zeitgemäßen Formen von Wirtschaftsdemokratie zu rufen? Bei der Suche nach Antworten lässt sich vielleicht von der Shareholder-Value-Bewegung lernen. Auch wertorientierte Steuerung und marktzentrierte Kontrolle haben sich nicht als Konzepte aus einem Guss und quasi über Nacht durchgesetzt. Wie gezeigt, handelte es sich um eine Vielzahl schrittweiser Veränderungen auf unterschiedlichen Ebenen, die sich schließlich zu einer sozialen Realität verdichtet haben, welche selbst durch Krisenprozesse epochaler Dimension nicht unbedingt zu erschüttern ist. Die potenziellen Träger einer wirtschaftsdemokratischen Bewegung verfügen derzeit über ungleich geringere Machtressourcen. Und sie besitzen nicht einmal kohärente Konzepte, um auf strategische Entscheidungen innerhalb wie außerhalb der Unternehmen Einfluss nehmen zu können. Doch schon die Tatsache, dass über Wirtschaftsdemokratie wieder geredet wird, verändert den Diskurs. Darin liegt, das zumindest lässt sich festhalten, eine Hoffnung für die Zukunft. 


\section{LITERATUR}

Aglietta, M. (2000): Ein neues Akkumulationsregime. Die Regulationstheorie auf dem Prüfstand, Hamburg

Aglietta, M./Rebérioux, A. (2005): Vom Finanzkapitalismus zur Wiederbelebung der sozialen Demokratie, Supplement der Zeitschrift Sozialismus 3 Arendt, H. (2006): Elemente und Ursprünge totalitärer Herrschaft. Antisemitismus, Imperialismus, totale Herrschaft, München

Becker, K. (2009): Die Bühne der Bonität. Wie mittelständische Unternehmen auf die neuen Anforderungen des Finanzmarkts reagieren, Berlin Bourdieu, P. (1998): Das ökonomische Feld, in: Der Einzige und sein Eigenheim. Schriften zu Politik \& Kultur, Hamburg, S. 162-204

Brinkmann, U. (2009): Markt, Unternehmenskultur und Beteiligung: Zum Wandel betrieblicher Kontroll- und Koordinationsmodi im Marktkapitalismus, Habilitationsschrift, Ms., Jena

Deutschmann, C. (1997): Die Mythenspirale. Eine wissenssoziologische Interpretation industrieller Rationalisierung, in: Soziale Welt 1, S. 55-70 Dörre, K. (2002): Kampf um Beteiligung. Arbeit, Partizipation und industrielle Beziehungen im flexiblen Kapitalismus, Opladen

Dörre, K. (2009): Die neue Landnahme. Dynamiken und Grenzen des Finanzmarktkapitalismus, in: Dörre, K./Lessenich, S./Rosa, H. (Hrsg.):

Soziologie - Kapitalismus - Kritik. Eine Debatte, Frankfurt a. M., S. 21-86 Dörre, K./Brinkmann, U. (2005): Finanzmarkt-Kapitalismus: Triebkraft eines flexiblen Produktionsmodells?, in: Windolf, P. (Hrsg.): FinanzmarktKapitalismus. Analysen zum Wandel von Produktionsregimen, Opladen, S. 58-84

Dörre, K./Holst, H./Nachtwey, O.: (2009). Organizing - A Strategic Option for Trade Union Renewal?, in: International Journal of Action Research 1, S. 33-67

Dörre, K./Röttger, B. (2006): Im Schatten der Globalisierung, Wiesbaden Fligstein, N. (2001): The Architecture of Markets: An Economic Sociology of Twenty-First-Century Capitalist Societies, Princeton

Friedman, M. (1984): Kapitalismus und Freiheit [1962], Frankfurt a. M.

Harvey, D. (2005): Der neue Imperialismus, Hamburg
Hiss, S. (2009): Möglichkeiten und Grenzen nachhaltiger Unternehmenssteuerung unter finanzmarktkapitalistischen Bedingungen?, Vortrag im Rahmen der Konferenz "Bringing capitalism back in - Arbeitssoziologie und Kapitalismustheorie" am 7./8. 10., Ms., Jena

Holst, H./Nachtwey, O./Dörre, K. (2009): Funktionswandel von Leiharbeit - Neue Nutzungsstrategien und ihre arbeits- und mitbestimmungspolitischen Folgen, Otto-Brenner-Stiftung (Hrsg.): Arbeitsheft 61, Frankfurt a. $M$.

Huber, B. (2009): „Wir werden jeder Regierung Druck machen“. Interview in Frankfurter Rundschau vom 25. September, S. 18-19

Kädtler, J. (2009): Finanzialisierung und Finanzmarktrationalität. Zur Bedeutung konventioneller Handlungsorientierungen im gegenwärtigen Kapitalismus, SOFI-Arbeitspapier 5, Göttingen

Lutz, B. (1984): Der kurze Traum immerwährender Prosperität. Eine Neuinterpretation der industriell-kapitalistischen Entwicklung im Europa des 20. Jahrhunderts, Frankfurt/New York

Luxemburg, R. (1975): Die Akkumulation des Kapitals. Ein Beitrag zur ökonomischen Erklärung des Imperialismus [1913], in: Dies.: Gesammelte Werke, Band 5, Berlin, S. 5-411

Merz, F. (2008): Mehr Kapitalismus wagen: Wege zu einer gerechten Gesellschaft, München

Rappaport, A. (1986): Creating shareholder value, New York Streeck, W. (2009): Re-Forming Capitalism. Institutional Change in the German Political Economy, Oxford

Urban, H.-J. (2009): „Der Finanzmarktkapitalismus ist stabiler, als wir dachten ", Interview in: Sozialismus 9, S. 31-36

Windolf, P. (2005): Was ist Finanzmarktkapitalismus?, in: Ders. (Hrsg.) Finanzmarktkapitalismus. Analyse zum Wandel von Produktionsregimen, Opladen, S. 20-57 